\title{
DEPRESIÓN POSPARTO EN EL EMBARAZO ADOLESCENTE: ANÁLISIS DEL PROBLEMA Y SUS CONSECUENCIAS
}

\author{
Claudia Wolff L. ${ }^{1,2}$, Paula Valenzuela X. ${ }^{3, a}$, Karim Esteffan S. ${ }^{1,4}$, Daniela Zapata B. 5 \\ ${ }^{1}$ Programa Magíster en Salud Pública, Escuela de Salud Pública, Facultad de Medicina, Universidad de Chile. ${ }^{2}$ Progra- \\ ma de Formación en Psiquiatría, Universidad Mayor. ${ }^{3}$ Escuela de Psicología, Universidad del Desarrollo. ${ }^{4}$ Programa \\ de Formación en Oftalmología, Universidad de los Andes. ${ }^{5}$ Hospital San Antonio, Putaendo, Servicio de Salud Acon- \\ cagua.
}

a Psicóloga

\section{RESUMEN}

El embarazo adolescente es un importante problema de Salud Pública, que se asocia a consecuencias biológicas, psicológicas y sociales negativas tanto para la madre como para el hijo. Uno de los riesgos frente a los que las madres adolescentes están particularmente vulnerables es a la depresión posparto (DPP). Existen extensas investigaciones sobre el fenómeno de la DPP, pero los estudios en madres adolescentes son limitados y suelen provenir de países desarrollados. En Chile no existen estudios publicados que se aboquen específicamente a la DPP en adolescentes. Por lo mismo, es que esta revisión pretende dar cuenta de los principales hallazgos internacionales y nacionales sobre prevalencia, factores de riesgo, consecuencias y abordaje de la DPP en general, y en madres adolescentes en particular, con el propósito de fortalecer el conocimiento de los profesionales que trabajan con estas jóvenes, ya sea en el área de salud mental, obstetricia o pediatría.

\section{PALABRAS CLAVE: Embarazo adolescente, depresión posparto}

\section{SUMMARY}

Adolescent pregnancy is an important public health issue associated with negative biological, psychologi$\mathrm{cal}$ and social consequences for the mother and the child. One of the main risks adolescent mothers are particularly vulnerable to postpartum depression. Literature on postpartum depression is broad, but studies regarding the phenomenon in adolescent mothers are scarce, and come mostly from developed countries. In Chile there are no published studies centred on postpartum depression in adolescents. Therefore, this article aims to describe the main international and national findings on the prevalence, risk factors, consequences and management of postpartum depression in general, and in adolescents in particular, with the goal of strengthening the knowledge of professionals caring for adolescent mothers in mental health, obstetric and pediatric services.

\section{KEY WORDS: Adolescent pregnancy, postpartum depression}




\section{INTRODUCCIÓN}

El embarazo adolescente es un importante problema de Salud Pública, que incide directamente en la posibilidad de las jóvenes de completar a cabalidad las tareas de la adolescencia y de asumir los desafíos que implica el embarazo y el nacimiento de un hijo. El embarazo en esta etapa de la vida se asocia a consecuencias biológicas, psicológicas y sociales negativas tanto para la madre como para el hijo; siendo la depresión posparto (DPP) uno de los principales riesgos $(1,2)$

A nivel internacional existe una amplia literatura respecto a la DPP. Numerosas investigaciones en países desarrollados (3-7) y en vías de desarrollo $(8,9)$, incluido Chile $(10,11)$, informan sobre la prevalencia del trastorno, los factores de riesgo y las consecuencias asociadas, así como de las intervenciones efectivas para tratarlo. A pesar de esto, son pocas las investigaciones que se han abocado a la búsqueda de factores de riesgo psicosocial, sintomatología depresiva y prevalencia de DPP exclusivamente en madres adolescentes (12-15). En Chile, si bien algunas investigaciones han abordado el tema de la salud mental entre las puérperas adolescentes, no existen estudios publicados cuyo objetivo central sea el estudio de la DPP (1).

El objetivo de este estudio es revisar los principales hallazgos en relación a la DPP en el contexto del embarazo adolescente.

\section{METODOLOGÍA}

Se realizó una selección de artículos publicados en las bases de datos PubMed, Scielo, SIBUC, utilizando como palabras clave para la búsqueda, "embarazo adolescente", "maternidad adolescente", "depresión en adolescentes" y "depresión posparto". Para la búsqueda se incluyeron artículos en idioma inglés y español, sin restricción por año de publicación. El criterio de selección implicó que los artículos contuvieran aspectos centrales para el tópico en desarrollo.

\section{RESULTADOS}

Características generales. Por convención, embarazo adolescente se define como todos aquellos embarazos que se presentan en mujeres menores de 19 años. Existe amplia variabilidad en la incidencia alrededor del mundo; la tasa de natalidad en jóvenes entre 15 y 19 años alcanza el $10 \%$ en Estados Unidos, el 4\% en Europa Occidental y Ja- pón $(16,17)$. En Chile en tanto, la fecundidad en menores de 19 años experimentó un ascenso sostenido entre la década del 80 y el año 2 mil, con una tasa actual de natalidad adolescente cercana al $15 \%(18-21)$.

El embarazo adolescente se ha asociado a bajo nivel socioeconómico y educacional, inicio precoz de la actividad sexual y a psicopatología, tal como la depresión (2). Asimismo, se sabe que las madres adolescentes tienen mayor riesgo de morbimortalidad obstétrica $(20,21)$, más probabilidad de deserción escolar, inactividad laboral y dependencia económica $(19,20,23)$, y a establecer vínculos de apego inseguros con sus hijos (2). Éstos, en tanto, estarían más expuestos a la morbimortalidad perinatal y neonatal, a la prematurez y bajo peso al nacer, al abuso y/o negligencia parental y a problemas conductuales y de rendimiento desde la etapa preescolar $(2,20,21)$.

La DPP, por su parte, se define como un episodio de ánimo depresivo y/o anhedonia, sentimientos de culpa, dificultad para tomar decisiones, y síntomas somáticos, tales como fatigabilidad, cambios en el apetito y en el patrón de sueño-vigilia, pudiendo aparecer ideación e intento suicida e incluso síntomas psicóticos (8). Se considera de etiología multifactorial y lo que distinguiría a este cuadro de otros episodios depresivos es su presentación en las primeras cuatro semanas del posparto, aunque varios autores consideran que puede iniciarse hasta un año o más después del parto $(10,24)$.

Los episodios depresivos en el posparto comparten las mismas características que en otros periodos de la vida, pudiendo eso sí, ser más frecuente el curso fluctuante y la labilidad emocional. Asimismo, es común que los sentimientos depresivos, ideas obsesivas y la culpa, giren en torno al recién nacido. La puérpera puede sentir que es mala madre e incapaz de cuidar bien a su hijo, o sentirse desinteresada por éste. Las ideas delirantes, cuando las hay, incluso pueden aludir a que el bebé está poseído, o que tiene poderes especiales (24). Los criterios para definir un episodio depresivo mayor se presentan en la Tabla I.

La prevalencia de DPP muestra gran variabilidad en el reporte, con valores entre 5 y $50 \%$, dependiendo fundamentalmente de la población y el periodo de tiempo estudiado, así como de los instrumentos utilizados (10). En aquellas investigaciones en que se han usado criterios operacionales estrictos para definir los casos de DPP, la cifra suele encontrarse alrededor del $10 \%$, equivalente a la prevalencia de depresión en mujeres no embarazadas (12). 
Tabla I

\title{
CRITERIOS PARA EL EPISODIO DEPRESIVO MAYOR SEGÚN EL MANUAL DIAGNÓSTICO Y ESTADÍSTICO DE LOS TRASTORNOS MENTALES DE LA SOCIEDAD AMERICANA DE PSIQUIATRÍA, DSM-IV (9)
}

\begin{abstract}
A. Presencia de cinco (o más) de los siguientes síntomas durante al menos 2 semanas, que presentan un cambio respecto a la actividad previa; uno de los síntomas debe ser estado de ánimo depresivo o pérdida de interés o de la capacidad de placer
\end{abstract}

1. Estado de ánimo depresivo la mayor parte del día, casi cada día según lo indica el propio sujeto o la observación realizada por otros

2. Disminución acusada del interés o la capacidad para el placer en todas o casi todas las actividades, la mayor parte del día, casi día a día (según refiere el propio sujeto u observan los demás)

3. Pérdida importante de peso sin hacer régimen, o aumento de peso, o pérdida o aumento del apetito casi cada día

4. Insomnio o hipersomnia casi cada día

5. Agitación o enlentecimiento psicomotor casi cada día (observable por los demás, no meras sensaciones de inquietud o de estar enlentecido)

6. Fatiga o pérdida de energía casi cada día

7. Sentimientos de inutilidad o culpa excesivos o inapropiados (que pueden ser delirantes) casi cada día (no los simples autoreproches o culpabilidad por el hecho de estar enfermo)

8. Disminución de la capacidad de pensar o concentrarse, o indecisión, casi cada día (ya sea una atribución subjetiva o una observación ajena)

9. Pensamientos recurrentes de muerte (no sólo temor a la muerte), ideación suicida recurrente sin un plan específico, o una tentativa o un plan específico para suicidarse

B. Los síntomas no cumplen criterios para un episodio mixto

C. Los síntomas provocan malestar clínicamente significativo o deterioro social, laboral o de otras áreas importantes de la actividad del individuo

D. Los síntomas no son debidos a los efectos fisiológicos directos de una sustancia (droga o medicamento), o una enfermedad médica

E. Los síntomas no se explican mejor por la presencia de un duelo, los síntomas persisten durante más de 2 meses, se caracterizan por una acusada incapacidad funcional, preocupaciones mórbidas de inutilidad, ideación suicida, síntomas psicóticos o enlentecimiento psicomotor

El instrumento más usado para medir DPP corresponde a la Escala de Depresión Posnatal de Edimburgo (EPDS), validada en múltiples países e idiomas, incluido Chile (25). Originalmente concebida para ser utilizada en el puerperio, en muchos países su uso se ha extendido al periodo prenatal y también a la evaluación de los padres (25). Otros instrumentos de autoreporte que también se utilizan frecuentemente son el Inventario de Depresión de Beck (BDI), la Escala de Depresión del Centro
Epidemiológico (CES-D) y la de Hamilton (Ham-D) (16).

En Estados Unidos, en un estudio con representatividad nacional, usando los criterios del DSM-IV, se encontró una prevalencia de depresión a los 12 meses posparto de 9,3\% (3). En el Reino Unido, se evaluaron 12 mil mujeres con el EPDS a las 8 semanas posparto, encontrándose una prevalencia de síntomas depresivos del 10\% (4). En Chile, Jadresic y cols (10), y Alvarado y cols (11), mediante 
entrevistas psiquiátricas estructuradas, encontraron una incidencia de DPP de 10,2 y $9,2 \%$, respectivamente.

Al igual a lo que ocurre con la estimación de DPP en puérperas en general, el reporte de DPP en adolescentes muestra importantes fluctuaciones, con valores que oscilan entre 20 y $57 \%$ (Tabla II). Logsdon y cols (13) y Cox y cols (14) aplicaron el CES-D a puérperas adolescentes, encontrando un $37 \%$ de síntomas de DPP entre las 4 y 6 semanas y un $53,6 \%$ al año postparto, respectivamente. Schimdt y cols (15), en tanto, siguió durante cuatro años a madres adolescentes con el BDI, observando un $36,7 \%$ de síntomas depresivos a los 3 meses posparto y $21,1 \%$ a los 48 meses.

Figuereido y cols en Portugal (26), comparó a puérperas adultas y adolescentes, encontrando una tasa de $9,3 \%$ en las primeras y $25,9 \%$ en las segundas. Piyasil (9), en Tailandia hizo esta misma comparación encontrando una prevalencia de DPP de 23 y 12,9\%, respectivamente.

Factores asociados: Varios factores han sido asociados a la DPP, irrestrictamente de la edad de la puérpera. Dentro de los antecedentes personales destacan como factores de riesgo la personalidad altamente neurótica, la tendencia a la introversión, los antecedentes psiquiátricos en la familia $(5,6)$ y los episodios depresivos previos, ya sea anteparto o en un posparto anterior. De hecho, presentar síntomas depresivos durante el embarazo se considera el principal factor de riesgo (12). Asimismo, el "postpartum blues" o disforia posparto, período de labilidad emocional que aparece entre los primeros 3 a 15 días posparto, y que se presenta en alrededor del $50 \%$ de las puérperas, también se ha asociado a la aparición de DPP (7).

Los eventos vitales estresantes en los 12 meses previos al parto, el estilo de apego inseguro (27), la falta de apoyo emocional y financiero por parte del marido o pareja (28), el no tener pareja (29), depresión en la pareja, bajo apoyo social de la familia o amigos (30), tener una mala relación con la propia madre, se han identificado como factores psicosociales asociado a DPP.

El embarazo no planificado o no deseado (8), la ideación o intento de aborto (31), los abortos espontáneos previos (32), los embarazos complicados o de alto riesgo (33), un bebé del sexo no deseado

Tabla II

PREVALENCIA DE DPP EN ADOLESCENTES, SEGÚN AUTOR, AÑO DE PUBLICACIÓN, INSTRUMENTO UTILIZADO, POBLACIÓN Y TAMAÑO MUESTRAL

\begin{tabular}{|c|c|c|c|c|c|}
\hline Autor & Año & $\begin{array}{l}\text { Instrumento } \\
\text { utilizado }\end{array}$ & $\begin{array}{l}\text { Población } \\
\text { estudiada }\end{array}$ & $\begin{array}{l}\text { Tamaño } \\
\text { muestral }\end{array}$ & Prevalencia \\
\hline Logsdon y cols. & 2008 & CES-D & $\begin{array}{l}\text { Adolescentes } 4-6 \\
\text { semanas posparto }\end{array}$ & 85 & $37 \%$ \\
\hline Cox y cols. & 2008 & CES-DC & $\begin{array}{l}\text { Adolescentes } \\
2 \text { semanas posparto }\end{array}$ & 168 & $53,6 \%$ \\
\hline Figuereido y cols. & 2007 & EPDS & $\begin{array}{l}\text { Adultas y adolescentes } \\
2-3 \text { meses posparto }\end{array}$ & 54 & $25,9 \%$ \\
\hline Schimdt y cols. & 2006 & BDI & $\begin{array}{l}\text { Adolescentes entre } 3 \\
\text { y } 48 \text { meses posparto }\end{array}$ & 623 & $\begin{array}{l}36,7 \% \text { (3 meses) } \\
21,1 \% \text { (48 meses) }\end{array}$ \\
\hline Barnet y cols. & 1996 & CES-DC & $\begin{array}{l}\text { Adolescentes } \\
2-4 \text { meses posparto }\end{array}$ & 125 & $32 \%$ (4 meses) \\
\hline Panzarine y cols. & 1995 & BDI & $\begin{array}{l}\text { Adolescentes } \\
6 \text { meses posparto }\end{array}$ & 50 & $24 \%$ (6 meses) \\
\hline
\end{tabular}

CES-D: Escala de depresión del Centro de Estudios Epidemiológicos. CES-DC: Escala de Depresión del Centro de Estudios Epidemiológicos para niños, versión corta. EPDS:Escala de Depresión posnatal de Edimburgo. BDI: Inventario de depresión de Beck. 
(8), o con alguna malformación congénita (34), y el no dar lactancia materna (29) son factores ginecoobstétricos identificados como factores de riesgo. El tipo de parto, en tanto, no ha sido relacionado a DPP (35).

La DPP se ha asociado también a varios efectos a corto y largo plazo sobre la interacción entre la madre y el niño. Existiría mayor interrupción de la lactancia materna exclusiva (36), menor desarrollo de las habilidades maternales y posibilidades de interacciones positivas entre la díada madre-hijo, así como más problemas cognitivos-conductuales en el niño $(3,37)$.

Varias investigaciones se han abocado a la búsqueda de factores de riesgo específicamente entre madres adolescentes. Tal como los estudios que han intentado ligar factores socioeconómicos con DPP, sin distinción por rango etáreo, en adolescentes los resultados no han sido concluyentes. Barnet y cols (38), evaluaron la influencia del nivel socioeconómico, sin encontrar una relación significativa entre éste y síntomas depresivos, mientras que Secco y cols (39), encontraron una relación estadísticamente significativa entre menor nivel socioeconómico y DPP en las madres adolescentes. Caldwell y cols (40) por su parte, estudiaron la asociación entre etnicidad y DPP, sin encontrar una diferencia significativa entre las adolescentes afroamericanas y las caucásicas.

La relación entre nivel educacional y DPP también ha mostrado resultados disímiles. Barnet y cols (38), diferenciaron entre las madres que habían abandonado el colegio y aquellas que seguían asistiendo a clases, sin encontrar diferencias en la prevalencia de DPP. Mientras que Kalil y cols (41), mostraron que aquellas jóvenes con deserción escolar, presentaban mayores índices de DPP a los 6 meses postparto.

En cuanto a la edad, Figuereido y cols (26), concluyeron que ser adolescente era un factor de riesgo independiente para DPP, mientras que Kalil y cols (41), reportaron mayor riesgo de DPP entre las jóvenes de 15 años respecto a las de 16 ó 17.

Respecto a los factores psicosociales, Logson y cols (13), encontraron que el principal factor predictor de DPP era el percibirse como estresada, en tanto que Barnet y cols (38), no sólo encontraron que el estrés aumentaba significativamente entre el tercer trimestre de embarazo y el posparto, sino que además se asociaba a la presencia de DPP. Lesser \& Koniak-Griffin (42), en tanto, encontraron que tener antecedentes de abuso físico y/o sexual en la infancia, predecía en forma estadísticamente significativa la aparición de DPP entre las madres adolescentes.
Respecto al apoyo social, Barnet y cols (38), concluyeron que recibir apoyo social por parte de la propia madre del y/o padre del bebé constituía un factor protector de DPP en adolescentes, mientras los conflictos con el progenitor del niño constituían un factor de riesgo. Caldwell y cols (43), también estudiaron la relación de la adolescente con su propia madre, encontrando que a mayor apoyo percibido por parte de esta última, menor era el puntaje obtenido en el CES-D. Kalil y cols (41), encontraron que a mayor conflictividad en el núcleo familiar, mayor era el puntaje de las adolescentes en el CES-D a los 6 meses posparto.

Panzarine y cols (44), reportaron que a mayor sintomatología depresiva al BDI, menor era la confianza de las adolescentes en sus habilidades maternales y menor su satisfacción con el rol materno. Birkeland y cols (45), observaron que a menor confianza en las propias competencias maternas y mayores preocupaciones respecto a la apariencia física y peso corporal, las adolescentes presentaban mayor sintomatología depresiva en las adolescentes. Secco y cols (39), concluyeron que el principal factor predictor de DPP en adolescentes a las $4 \mathrm{se}$ manas posparto era el tener emociones negativas hacia el cuidado del bebé durante el embarazo.

En cuanto a las consecuencias de la DPP, Barnet y cols (46) en un estudio prospectivo, mostraron que las jóvenes deprimidas tenían un riesgo relativo de 1,44 en relación a las no deprimidas de presentar un segundo embarazo entre los 12 y 24 meses posparto. Respecto a la relación madre-hijo, Panzarine y cols (44), reportaron una asociación estadísticamente significativa entre síntomas depresivos y peor interacción de las madres adolescentes con sus bebés al momento de alimentarlos; mientras que Field y cols (47), concluyeron que bebés de adolescentes deprimidas, mostraban menor talla, menor circunferencia craneana y mayor morbilidad entre los 3 y 6 meses de edad.

Leadbeater y cols (48), evaluaron la calidad de las interacciones madre adolescente-hijo, encontrando que a mayor sintomatología depresiva durante el primer año posparto, existían menos interacciones recíprocas positivas entre la díada a los 20 meses. Al medir a los mismos niños, en la etapa preescolar, vieron que aquellos con madres deprimidas en el posparto presentaban más problemas conductuales. Asimismo, Lesser \& Koniak-Griffin (42), encontraron una correlación negativa entre síntomas depresivos y la calidad de la interacción diádica entre las 4 y 6 semanas posparto.

Manejo: En la práctica clínica, la DPP suele ser subdiagnosticada y subtratada. Las razones por las que esto ocurre son variadas, pero se plantea que 
las expectativas sociales y familiares que recaen sobre las puérperas las hacen reticentes a revelar sus problemas anímicos. De hecho, un estudio mostró que del total de mujeres con DPP, tan solo $30 \%$ pensaba que podía estar sufriendo un episodio depresivo, y $80 \%$ no había consultado al equipo de salud por su sintomatología (49). Entre las madres adolescentes la DPP también suele pasar desapercibida, y las barreras para la identificación y tratamiento en ellas han sido prácticamente inexploradas (50).

El traslape sintomático que se produce entre los síntomas depresivos y los puerperales también contribuyen a dificultar el diagnóstico. Las alteraciones neurovegetativas y neurocognitivas características de la depresión, tales como fatiga, dificultad para dormir, labilidad emocional, dificultad para concentrarse y baja libido, son también muy frecuentes de encontrar entre las puérperas (51). Por lo mismo, es muy importante indagar respecto al origen de los síntomas. Útil resulta discriminar la fatiga física de la "emocional", en donde lo que predomina es la anhedonia. Asimismo, el insomnio, en el que la madre no logra conciliar el sueño mientras el bebe duerme también orienta a DPP (52).

En el caso de las jóvenes, los síntomas depresivos suelen acompañarse de irritabilidad, por lo que su presencia en una puérpera adolescente debe hacer sospechar de DPP. De hecho, Shanok \& Miller (53), encontraron que tanto la tristeza como la irritabilidad, eran los principales síntomas descritos por las madres adolescentes deprimidas.

Por otro lado, la DPP suele ser subdiagnosticada porque en el control de salud pre y posnatal generalmente no está contemplada la búsqueda de factores de riesgo ni de síntomas de depresión. En Estados Unidos, por ejemplo, el tamizaje de DPP no es una medida estándar a nivel nacional (52). En nuestro país, en los controles prenatales se evalúan factores de riesgo psicosocial en la madre, considerándose todo embarazo adolescente como de alto riesgo, y se aplica de rutina el EPDS en el puerperio. Por el momento, sin embargo, estas intervenciones sólo contemplan a los niveles socioeconómicos más bajos atendidos en el sistema público de salud (54).

El tratamiento de la DPP se basa en los mismos principios que en la población general. Un enfoque biopsicosocial permite cubrir todos los factores involucrados así como optimizar la respuesta clínica. La gravedad de los síntomas determinará la modalidad terapéutica a utilizar, considerando que los mejores resultados se obtienen del manejo combinado de los factores biológicos, psicológicos y sociales.

Los factores biológicos se manejan fundamen- talmente a través de la promoción del descanso y el buen dormir y de la farmacoterapia, cuya elección depende fundamentalmente de los antecedentes depresivos previos, y si la madre está o no lactando. La terapia hormonal, de luz e incluso terapia electroconvulsiva, pueden ser necesarias en caso que los síntomas no remitan a pesar del uso correcto de los fármacos antidepresivos $(52,55)$.

Para los factores psicológicos es útil la psicoterapia, individual o grupal, que en muchos casos deberá incluir a la pareja o familia de la puérpera. La terapia de grupo permite trabajar en la psicoeducación, fomentar el apoyo entre mujeres que se encuentran viviendo situaciones similares, así como explorar la red social y comunitaria, ya que en muchos casos se requiere la derivación a servicios de asistencia social y/o judiciales específicos (56).

\section{CONCLUSIONES}

La DPP es un importante problema de salud pública que afecta a puérperas de todas las edades, asociándose a factores de riesgo tales como, depresión previa, malas relaciones interpersonales dentro de la familia, con la pareja o padre del bebé y bajo apoyo social. Asimismo, la DPP se asocia a alteraciones en el vínculo madre-hijo, con menor desarrollo y más problemas conductuales en el niño.

Entre las madres adolescentes, la DPP es especialmente frecuente, y en ellas, además de los factores ya descritos, se agregan algunos particulares, tales como el riesgo de deserción escolar, de abuso y negligencia hacia el niño y mayor probabilidad de un segundo embarazo adolescente.

Existe escasa información respecto a los factores específicos que inciden en el diagnóstico y tratamiento de la DPP en adolescentes, por lo mismo, las investigaciones debieran orientarse en esa línea. En Chile no contamos con información específica para este subgrupo, por lo que es imperativo que se trabaje en esta área, especialmente importante es la prevalencia de DPP entre las madres adolescentes, ya que no conocemos la magnitud del problema a nivel nacional. Por otro lado, es importante evaluar la eficacia de los programas implementados en el sistema público de salud para el manejo de los factores de riesgo psicosociales en embarazadas y de pesquisa de DPP.

Urge que los profesionales de la salud nacional, tanto del sistema público como privado que trabajan con embarazadas, y especialmente aquellos que trabajan con adolescentes, actualicen sus conocimientos respecto al diagnóstico y tratamiento de la DPP. Es importante incorporar a la rutina de 
trabajo, la búsqueda activa de síntomas depresivos y de factores de riesgo para DPP, idealmente desde el periodo prenatal. Para esto, lo más conveniente es la capacitación en el uso de algún instrumento de pesquisa de DPP, tal como el EPDS, validado en nuestro país y ampliamente utilizado en adolescentes.

\section{BIBLIOGRAFÍA}

1. Vera G, Gallegos M, Varela M. Salud mental y embarazo en adolescentes. Contexto de la gestación y consecuencias biográficas tempranas. Rev Méd Chile 1999;127(4):437-43.

2. Reid V, Meadows-Oliver M. Postpartum depression in adolescent mothers: an integrative review of the literature. J Pediatr Health Care 2007;21(5):289-98.

3. Vesga-López O, Blanco C, Keyes K, Olfson M, Grant B, Hasin D. Psychiatric disorders in pregnant and postpartum women in the United States. Arch Gen Psychiatry 2008;65(7):805-15.

4. Ramchandani P, Stein A, Evans J, O'Connor T; ALSPAC Study Team. Paternal depression in the postnatal period and child development: a prospective population study. Lancet 2005;365(9478):2201-5.

5. Verkerk G, Denollet J, Van Heck G, Van Son M, Pop $V$. Personality factors as determinants of depression in postpartum women: a prospective 1-year follow-up study. Psychosom Med 2005;67(4):632-7.

6. Brugha T, Sharp H, Cooper S, Weisender C, Britto D, Shinkwin R, et al. The Leicester 500 Project. Social support and the development of postnatal depressive symptoms, a prospective cohort survey. Psychol Med 1998;28(1):63-79.

7. O'Hara M, Schlecht J, Lewis D, Wright E. Prospective study of postpartum blues. Biologic and psychosocial factors. Arch Gen Psychiatry 1991;48(9):801-6.

8. Gomes da Silva I, Tavares R, Azevedo R, Lessa B, Rosa P, Duarte A. Prevalence of postpartum depression and associated factors. Rev Saúde Pública 2006;40(1):1-6.

9. Piyasil V. Anxiety and depression in teenage mothers: a comparative study. J Med Assoc Thai 1998;81(2):1259.

10. Jadresic E, Nguyen D, Halbreich U. (2006). What does Chilean research tells us about postpartum depression (PPD)? J Affect Dissord 2007;102(1-3):23743.

11. Alvarado R, Rojas M, Monardes J, Neves E, Olea E, Perucca E, et al. Cuadros depresivos en el postparto y variables asociadas en una cohorte de 125 mujeres embarazadas. Rev Psiquiatr 1992;3(4):1168-76.

12. McElderry D. Screening of adolescent females for depression before, during and after pregnancy. J Pediatr Adolesc Gynecol 2001;14 (3),131-3.

13. Logsdon M, Hertweck $P$, Ziegler C, Pinto-Foltz. Testing a bioecological model to examine social support in postpartum adolescents. J Nurs Scholarsh 2008;40(2):116-23.

14. Cox J, Buman M, Valenzuela J, Pierre Joseph N, Mitchell A, Woods E. Depression, parenting attribute, and social support among adolescent mothers attending a teen tot program. J Pediatr Adolesc Gynecol 2008;21(5):275-81.

15. Schmidt R, Wiemann C, Rickert V, Smith E. Moderate to severe depressive symptoms among adolescent mothers followed four years postpartum. J Adolesc Health 2006;38(6):712-8.

16. Henshaw S. US teenage pregnancy statistics: with comparative statistics for women aged 20-24. New York, The Alan Guttmacher Institute, 1999.

17. Singh S, Darroch J. Adolescent Pregnancy and childbearing: levels and trends in developed countries. Fam Plann Perspec 2000;32(1):14-23.

18. MINSAL, DEIS. Hallado en: www.minsal.cl. Acceso: 20/10/2008.

19. Molina R, Molina T, González E. Madres niñas-adolescentes de 14 años y menos. Un grave problema de salud pública no resuelto en Chile. Rev Med Chile 2007;135(1):79-86.

20. Molina M, Ferrada C, Pérez R, Cid L, Casanueva V, García A. Embarazo en la adolescencia y su relación con la deserción escolar. Rev Med Chile 2004;132(1): 65-70.

21. Donoso E, Becker J, Villarroel L. Natalidad y riesgo reproductivo en adolescentes de Chile, 1990-1999. Rev Panam Salud Pública 2003;14(1): 3-8.

22. Logsdon M. Depression in adolescent girls: screening and treatment strategies for primary care providers. J Am Med Womens Assoc 2004;59(2):101-6.

23. Enfoques estadísticos, №9. Fecundidad. Octubre 2000. Boletín informativo del Instituto Nacional de Estadísticas de Chile. Hallado en: http://www.ine. cl/canales/sala_prensa/archivo_documentos/enfoques/2000/files/enfoque9.pdf . Acceso: 03/06/09.

24. Rush A, Keller M, Bauer M, Dunner D, Frank E, Klein D (Grupo de trabajo para los trastornos del estado del ánimo). Trastornos del estado del ánimo. En: Pichot, López-Ibor, Valdés (Editores). Manual diagnóstico y estadístico de los trastornos mentales (DSM IV), Masson SA, Barcelona, 1995.

25. Jadresic E, Araya R, Jara C. Validation of the Edinburgh Postnatal Depression Scales (EPDS) in Chilean postpartum women. J Psychosom Obstet Ginaecol 1995;16(4):187-91.

26. Figueiredo B, Pacheco A, Costa R. Depression during pregnancy and the postpartum period in adolescent and adult Portuguese mothers. Arch Womens Ment Health 2007;10(3):103-9.

27. Figueireido B, Bifulco A, Pacheco A, Costa R, Magarinho $R$. Teenage pregnancy, attachment style, and depression: a comparison of teenage and adult pregnancy women in a Portuguese series. Attach Hum Dev 2006;8 (2),123-38.

28. Wilson L, Reid A, Midmer D, Biringer A, Carroll J, Stewart D. Antenatal psychosocial risk factors associated with adverse postpartum family outcomes. CMAJ 1996;154(6):785- 99.

29. Yonkers K, Ramin S, Rush A, Navarrete C, Carmody $\mathrm{T}$, March $\mathrm{D}$, et al. Onset and persistence of postpartum depression in an inner-city maternal health clinic system. Am J Psychiatry 2001;158(11):1856- 63.

30. Murray D, Cox J, Chapman G, Jones P. Childbirth: life 
event or start of a long-term difficulty? Further data from the Stoke-on-Trent controlled study of postnatal depression. Br J Psychiatry 1995;166(5):595-600.

31. Kumar R, Robson K. A prospective study of emotional disorders in childbearing women. Br J Psychiatry 1984;144:35-47.

32. Cryan E, Keogh F, Connolly E, Cody S, Quinlan A, Daly I. Depression among postnatal women in an urban Irish community. Ir J Psych Med 2001;18(1):510.

33. Josefsson A, Angelsiöö L, Berg G, Ekström C, Gunnervik $C$, Nordin $C$, et al. Obstetric, somatic, and demographic risk factors for postpartum depressive symptoms. Obstet Gynecol 2002;99(2):223-8.

34. Rona R, Smeeton N, Beech R, Barnett A, Sharland G. Anxiety and depression in mothers related to severe malformation of the heart of the child and foetus. Acta Paediatr 1998;87(2):201-5.

35. Patel R, Murphy D, Peters T. Operative delivery and postnatal depression: a cohort study. BMJ 2005;330(7496):879. doi:10.1136/bmj.38376.603426. D3

36. Hasselman M, Werneck G, Cardim da Silva C. Symptoms of postpartum depression and early interruption of exclusive breastfeeding in the first two months of life. Cad Saúde Pública 2008;24(S2):S341S52.

37. Poobalan A, Aucott L, Ross L, Smith W., Helms P, Williams J. Effects of treating postnatal depression on mother-infant interaction and child development. $\mathrm{Br} \mathrm{J}$ Psychiatry 2007;91:78-86.

38. Barnet B, Duggan A, Wilson,M, Joffe A. Association between postpartum substance use and depressive symptoms, stress, and social support in adolescent mothers. Pediatrics 1995;96(4 Pt 1): 659-66.

39. Secco M, Profit S, Kennedy E, Walsh A, Letorneau $\mathrm{N}$, Steward M. Factors affecting postpartum depressive symptoms of adolescent mothers. JOGNN 2007,36(1):47-54.

40. Caldwell C, Antonucci H, Jackson J, Wolford M, Osofsky D. Perceptions of parental support and depressive symptomatology among black and white adolescent mothers. J Emotional Behavioural Disorders 1997;5(3):173-83.

41. Kalil A, Spencer M, Spieker S, Gilchrist L. Effects of grand-mother coresidence and quality of family relationships on depressive symptoms in adolescent mothers. Family Relations 1998;47(4):433-41.

42. Lesser J, Koniak-Griffin D. The impact of physical abuse on chronic depression in adolescent mothers. J Pediatr Nurs 2000,15(6):378-87.

43. Caldwell C, Antonucci H, Jackson J. Supportive/conflictual family relations and depressive symptomatology: Teenage mother and grandmother perspectives. Family Relations 1998;47(4):395-402.

44. Panzarine S, Slater E, Sharps P. Coping, social support, and depressive symptoms in adolescent mothers. J Adolesc Health 1995;17(2):113-99.

45. Birkeland R, Thompson J, Phares V. Adolescent motherhood and postpartum depression. J Clin Child Adolesc Psychol 2005;34(2):292-300.

46. Barnet B, Liu J, Devoe M. Double jeopardy: depressive symptoms and rapid subsequent pregnancy in adolescent mothers. Arch Pediatr Adolesc Med 2008;162(3):246-52.

47. Field T, Pickens J, Prodromidis M, Malphurs J, Fox N, Bendell D, et al. Targeting adolescent mothers with depressive symptoms for early intervention. Adolescence 2000;35(138):381-414.

48. Leadbeater B, Bishop S, Raver C. Quality of mothertoddler interactions, maternal depressive symptoms, and behaviour problems in preschoolers of adolescent mothers. Dev Psychol 1996;32(2):280-8.

49. Whitton A, Warner R, Appleby L. The pathway to care in post-natal depression: women's attitudes to postnatal depression and its treatment. $\mathrm{Br} \mathrm{J}$ Gen Pract 1996;46(408):427-8.

50. Logsdon MC, Usui W, Pinto-Foltz M, Rakestraw VL. Intention to seek depression treatment in adolescent mothers and a comparison group of adolescent girls. Arch Psychiatr Nurs 2009;23(1):41-9.

51. Evans J, Heron J, Francomb, H, Oke, S, Golding J. Cohort study of depressed mood during pregnancy and after childbirth. BMJ 2001;323(7307):257-60.

52. Horowitz A, Goodman J. Identifying and treating postpartum depression. JGONN 2005;34(2):264-273.

53. Shanok A, Miller L.Depression and treatment with inner city pregnant and parenting teens. Arch Womens Ment Health 2007;10(5):199-210.

54. Chile Crece Contigo. Hallado en: http://www.crececontigo.cl/adultos/. Acceso: 20/10/2008.

55. Appleby L, Warner R, Whitton A, Faragher B. A controlled study of fluoxetine and cognitive-behavioral counselling in the treatment of postnatal depression. BMJ 1997;314 7085):932-6.

56. Meager I, Milgrom J. Group treatment for postpartum depression: a pilot study. Aust N Z J Psychiatry 1996;30(6):852-60. 